ARTICLE

DOI: $10.1038 / s 41467-017-00219-x$

OPEN

\title{
Compositionally-distinct ultra-low velocity zones on Earth's core-mantle boundary
}

\author{
Mingming Li il ${ }^{1}$, Allen K. McNamara², Edward J. Garnero ${ }^{1} \&$ Shule Yu ${ }^{1}$
}

The Earth's lowermost mantle large low velocity provinces are accompanied by small-scale ultralow velocity zones in localized regions on the core-mantle boundary. Large low velocity provinces are hypothesized to be caused by large-scale compositional heterogeneity (i.e., thermochemical piles). The origin of ultralow velocity zones, however, remains elusive. Here we perform three-dimensional geodynamical calculations to show that the current locations and shapes of ultralow velocity zones are related to their cause. We find that the hottest lowermost mantle regions are commonly located well within the interiors of thermochemical piles. In contrast, accumulations of ultradense compositionally distinct material occur as discontinuous patches along the margins of thermochemical piles and have asymmetrical cross-sectional shape. Furthermore, the lateral morphology of these patches provides insight into mantle flow directions and long-term stability. The global distribution and large variations of morphology of ultralow velocity zones validate a compositionally distinct origin for most ultralow velocity zones.

\footnotetext{
${ }^{1}$ Arizona State University, School of Earth and Space Exploration, PO Box 871404, Tempe, AZ 85287-1404, USA. ${ }^{2}$ Michigan State University, Department of Earth and Environmental Sciences, Natural Science Building, East Lansing, MI 48824, USA. Correspondence and requests for materials should be addressed to M.L. (email: Mingming.Li@asu.edu)
} 
U ltralow velocity zones (ULVZs) are mapped as geographically isolated zones of seismic anomalies detected on the core-mantle boundary $(\mathrm{CMB})^{1,2}$ with a significant reduction of seismic velocity (up to $10 \%$ for $\mathrm{P}$-wave and $30 \%$ for S-wave velocities) and sometimes increased density 3,4 . Mapped as thin $(5-40 \mathrm{~km})$ and relatively small (e.g., hundreds of kilometer laterally, or less ${ }^{2,4-6}$, but sometimes up to $1000 \mathrm{~km}$ long ${ }^{7}{ }^{8}$ ), ULVZs are more commonly found within or near the large low velocity provinces (LLVPs) ${ }^{9}$. For this reason, along with the predominance of the S-velocity $(V s)$ drops being up to three times that of $\mathrm{P}$-velocity $(V p)$ reductions, the ultralow wave speeds have been attributed to partial melt due to being in the hottest lowermost mantle regions ${ }^{2,4}$.

At odds with the solely partial melt hypothesis is that some seismic studies identify ULVZs well outside of the seismically observed LLVPs ${ }^{10-13}$ including beneath subduction regions?, where temperatures are assumed to be far lower than in the presumed upwelling regions of LLVPs. Furthermore, some ULVZs do not have $V$ s reduction substantially greater than their $V \mathrm{p}$ reduction ${ }^{14}$. Hypotheses other than solely partial melt may thus be necessary. A number of hypotheses have been proposed, including iron-enriched $(\mathrm{Mg}, \mathrm{Fe}) \mathrm{O}^{15}, 16$, iron-enriched post-perovskite $^{17}$ (recent geodynamic modeling results show that patches of post-perovskite can be temporarily stable within LLVPs $^{18}$ ), subducted banded iron formations ${ }^{19}$, subducted oceanic crust ${ }^{20}$ or other slab-derived materials ${ }^{21,22}$, and products of chemical reactions between the silicate mantle and Fe-rich core $^{23,24}$. While these possibilities (as well as partial melt) may all be viable, their relationship to deep mantle flow, especially in regards to being swept towards upwelling regions and their geometrical relationship to LLVPs remains unknown. Of particular interest is the thermochemical pile hypothesis to explain LLVPs, whereby dense basal material is swept into piles to explain the seismically observed LLVPs ${ }^{25-27}$. Two fundamental questions are: where are the highest temperatures inside LLVPs, and are they different from accumulation locations of any additional, ultradense material that may reside at the base of the mantle? Understanding the dynamics, destinations, and morphologies of ULVZs caused by a compositionally distinct vs. partial melt origin is necessary to provide a meaningful framework for the distribution of seismic observations. We thus carried out very high resolution, three-dimensional thermochemical numerical convection calculations to study the distribution and morphology of ULVZs.

Here we explore ULVZs attributed to ultradense, compositionally distinct material, as well as ULVZs attributed to melting in the hottest deep mantle. We find that the hottest lowermost mantle regions, where partial melting could occur to explain ULVZs, are located well within the interiors of thermochemical piles. In contrast, accumulation of ultradense compositionally distinct material occurs as discontinuous patches along the margins of thermochemical piles and have an asymmetrical cross-sectional shape. The origin of ULVZs, therefore, can be constrained from their locations and shapes. The global distribution and large variations of morphology of the seismologically observed ULVZs indicate a compositionally distinct a
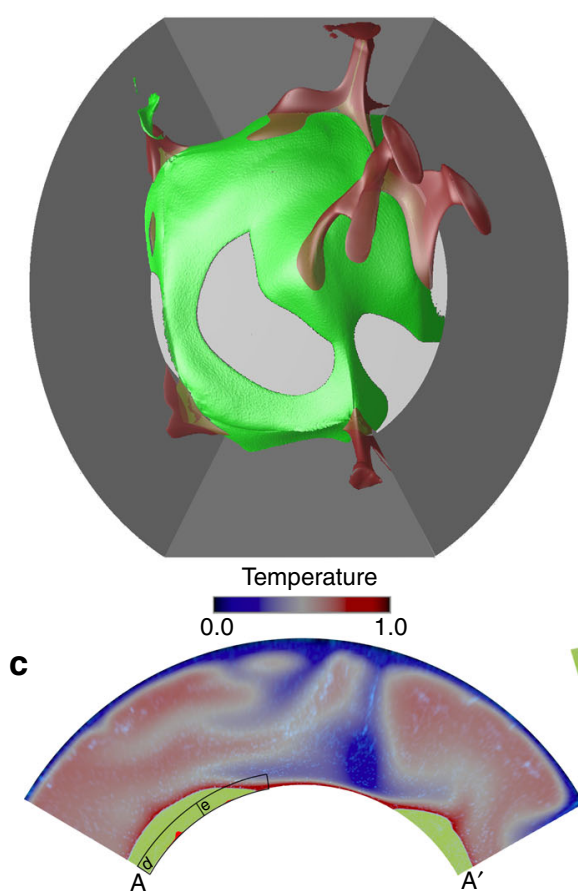

b

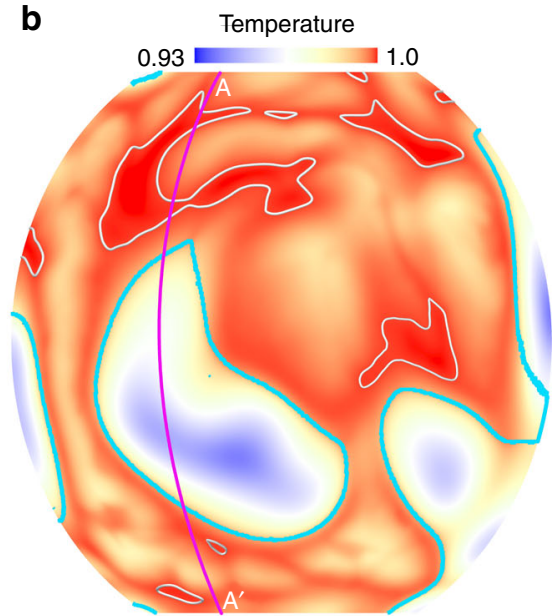

d

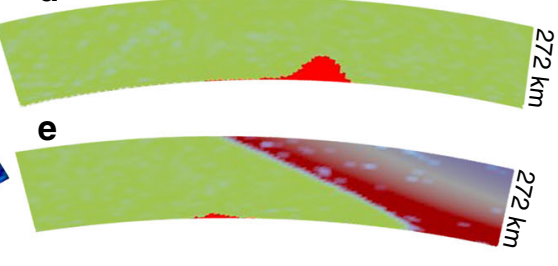

Fig. 1 Morphology and distribution of ULVZs caused by partial melting. a Top view of the model with mantle plumes (red isosurface of temperature of 0.683) forming on tops of thermochemical piles (green isosurface). The gray side boundaries define the domain of the 3D partial spherical geometry of the model, which spans $120^{\circ}$ in both longitudal and colatitudal directions. The downwellings (not shown) sweep pile material away, exposing the core (light gray) in these regions. b Temperature field in map-view at $5 \mathrm{~km}$ above the CMB. Light gray contours at $T=0.999$ show the hottest $10 \%$ regions of the piles by area. Thick cyan lines show the edges of piles. c Cross-section at locations marked by magenta line in Fig. 1b. The temperature field is shown by dark blue to red color, the thermochemical piles are shown in green color in the lowermost mantle, and the small red patches within the piles at the bottom of the mantle show the hottest regions. d, e Zoomed-in at the regions outlined by black boxes in Fig. 1c. The hottest regions (red patches inside the pile) are candidate regions for melting, and we identify the hottest regions where temperature is higher than mantle solidus. At $5 \mathrm{~km}$ above CMB, the hottest $10 \%$ pile regions by area are identified as candidate regions for melting by assuming a mantle solidus of $T=0.999$ (non-dimensional) at this depth. For other depths, the mantle solidus decreases with decreasing depth with a rate of $\sim 0.8 \mathrm{~K} \mathrm{~km}^{-138}$. All panels a-e are shown at $218 \mathrm{Myr}$ 
origin for most ULVZs, and that Earth's lowermost mantle contains small-scale compositional heterogeneities with elevated intrinsic density. ULVZs within LLVPs, however, might be explained by partial melting alone.

\section{Results}

Description of mantle convection models. Our reference model includes thermochemical piles, motivated by the multiple lines of evidence arguing a chemically distinct origin of LLVPs ${ }^{28-35}$. The conservation equations of mass, momentum and energy are solved using our modified version of the code CitcomCU ${ }^{36}$ in the Boussinesq approximation (Methods). We employ a Rayleigh number $\mathrm{Ra}=9.8 \times 10^{6}$ for most cases (Supplementary Table 1, using mantle thickness as the length-scale for non-dimensionalization). A $50 \times$ viscosity increase is employed from the upper mantle to the lower mantle (Supplementary Fig. 1). The temperature-dependent part of the viscosity is expressed as $\eta_{\mathrm{T}}=\exp [A(0.6-T)]$, where $T$ is non-dimensional temperature, and we use a non-dimensional activation coefficient of $A=9.21$ for most cases (Supplementary Table 1), leading to a $10000 \times$ viscosity range across the mantle due to changes in temperature. We employ a three-dimensional, partial-sphere geometry (Fig. 1a) in which the longitude and colatitude span $120^{\circ}$, and the dimensionless radius ranges from 0.55 to 1.0 (thus, from the $\mathrm{CMB}$ to the surface). We utilize 512, 512 and 128 elements in longitudinal, colatitudinal, and radial directions, respectively. The mesh is refined with depth resulting in a resolution of $5 \mathrm{~km}$ radially and $\sim 14.5 \mathrm{~km}$ laterally near the CMB. All boundaries are free-slip, isothermal at top and bottom, and insulating along the sides. The models are heated both from below and internally with a non-dimensional heat production rate of $H=60$ (using Earth's radius as the length-scale for non-dimensionalization).

We developed a hybrid tracer scheme to track composition (Methods), that simultaneously employs both ratio and absolute tracing methods ${ }^{37}$. The background mantle and the thermochemical piles are modeled with $\sim 710$ million ratio tracers and the ultradense ULVZ material is modeled with $\sim 50-110$ million absolute tracers, depending on the volume of ULVZ material (Supplementary Table 1). The hybrid tracer method more efficiently computes the advection of multi-scale composition, including both large-scale thermochemical piles and much smaller-scale accumulations of ultradense materials. The intrinsic density anomaly $(\Delta \rho)$ of each compositional component is non-dimensionalized as compositional buoyancy number $B$. The effective intrinsic density of each element is calculated by averaging the densities of each component, leading to an "effective buoyancy ratio", $B^{\text {eff. }}$. To construct an initial condition, we carry out a calculation with two compositional components (background mantle and thermochemical pile material) and we use the quasi-steady state temperature and composition field as initial condition for models in this study.

We perform 2 types of experiments, both of which include thermochemical piles to represent LLVPs. In the first set of experiments, we explore the positions and shapes of ULVZs caused by partial melting in the hottest mantle regions. In other words, we examine the morphology of the hottest lowermost mantle regions. In the second set of experiments, we explore the positions and shapes of ULVZs caused by the accumulation of the ultradense compositional component. We then examine the morphology of these accumulations.

ULVZs caused purely by partial melting. We first examine the locations of ULVZs due to partial melting alone (Case 1). The amount of partial melting above the $\mathrm{CMB}$ is controlled by the solidus temperature, liquidus temperature, and the mantle temperature above the CMB. The solidus temperature and liquidus temperature of a synthetic sample with chondritic-type composition at $\mathrm{CMB}$ pressure were measured by previous mineral physics experiments to be $\sim 4150$ and $\sim 4725 \mathrm{~K}^{38}$, respectively, and the solidus and liquidus temperatures for a natural fertile peridotite at $\mathrm{CMB}$ pressure were measured to be $\sim 4180$ and $5375 \mathrm{~K}$, respectively ${ }^{39}$. However, the solidus temperature for a pyrolitic composition with $\sim 400$ p.p.m. $\mathrm{H}_{2} \mathrm{O}$ has been reported to be as low as $\sim 3570 \mathrm{~K}^{40}$. Largely due to our limited knowledge about the lowermost mantle composition such as the amount of $\mathrm{H}_{2} \mathrm{O}$, the solidus temperature and liquidus temperature near the $\mathrm{CMB}$ pressure are not well constrained. In addition, there is large uncertainty of the CMB temperature, which has been suggested to be in the range of from $\sim 2500-2800 \mathrm{~K}$ to $\sim 3300-4300 \mathrm{~K}^{41}$, and the temperature of the thermal boundary layer above the $\mathrm{CMB}$ is poorly constrained. Our geodynamic models are non-dimensionalized and therefore do not independently constrain the absolute value of dimensional temperature. To convert non-dimensional to dimension temperature requires a choice for $\mathrm{CMB}$ temperature, which is not well constrained by observations. Because of these uncertainties, it becomes impractical to determine the amount of partial melting above the $\mathrm{CMB}$ in our models by comparing the dimensional lowermost mantle temperature in our models with the solidus and liquidus temperature at the $\mathrm{CMB}$ pressure measured in previous mineral physics experiments. Nonetheless, if there are ULVZs above the $\mathrm{CMB}$ caused by partial melting alone, they most likely exist in the hottest regions in the lowermost mantle. We thus focus on examining the location of hottest regions in the lowermost mantle in Case 1.

Case 1 includes 2 compositions: background mantle and piles with a buoyancy number $B_{\mathrm{p}}=0.8$ (or $3.6 \%$ denser than the background mantle if scaled using reference temperature and thermal expansivity as given in Supplementary Table 2). Figure 1 shows a snapshot at $218 \mathrm{Myr}$. In this study, the geological time is scaled by the transit time and we assume that one transit time (the time it takes for a slab to descend from surface to the CMB) equals to $60 \mathrm{Myrs}^{42,43}$. Figure 1a illustrates thermochemical piles (green) with mantle plumes (red) rising from cusps along their tops. Figure $1 \mathrm{~b}$ shows the temperature field at $5 \mathrm{~km}$ height above the $\mathrm{CMB}$, in which it is observed that the hottest $10 \%$ regions of the piles by area (marked by light gray contours of $T=0.999$ ) occur within pile interiors, well inward from their edges (pile edges are outlined by cyan lines). The dimensional temperature for the hottest $10 \%$ regions at this depth is in the range of $\sim 3600-4000 \mathrm{~K}$, if dimensionalized with a reference potential temperature of $\Delta T=2500 \mathrm{~K}$ (Supplementary Table 2), after adding an adiabatic temperature increase from the surface (with a temperature of $273 \mathrm{~K}$ ) down to $5 \mathrm{~km}$ above the $\mathrm{CMB}$ with an adiabatic thermal gradient of $0.3-0.4 \mathrm{~K} \mathrm{~km}^{-1}$. Interestingly, these hottest regions have a temperature comparable to the solidus temperature of a pyrolitic or chondritic composition near the $\mathrm{CMB}$ pressure, depending on the $\mathrm{H}_{2} \mathrm{O}$ content in the lowermost mantle ${ }^{38-40}$. However, it needs to be emphasized that the dimensional temperature in our models depends on the choice of reference temperature for scaling. We thus focus on the location of hottest regions that are the best candidate locations for partial melting.

We plot the hottest regions in the cross-section shown in Fig. 1c, with zoom-ins shown in Fig. 1d, e. The hottest regions reside within and with some distance from the pile edges because of cooling of thermochemical pile margins by the cooler, surrounding non-pile mantle (Supplementary Note 1; Supplementary Fig. 2). We find the hottest regions occur well within the interior of thermochemical piles throughout the model run 


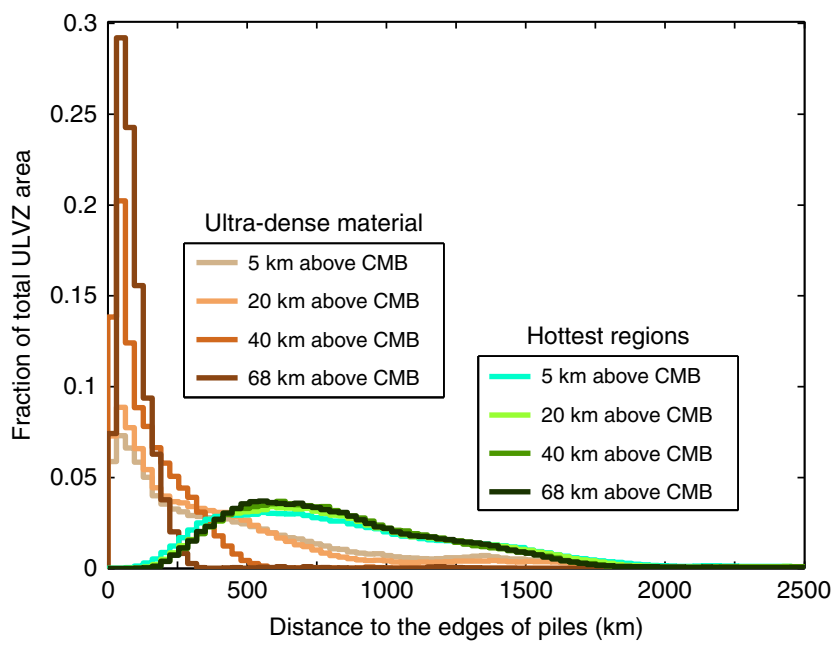

Fig. 2 Distance of ULVZs from thermochemical pile edges. For each distance in the horizontal axis, the corresponding vertical axis shows the areal fraction of hottest regions or accumulations of ultradense material at this distance. Here, the hottest regions refer to candidate regions for melting (e.g., Fig. 1b-e), the regions with accumulations of ultradense material refer to places where the effective buoyancy ratio $\left(B^{\text {eff }}\right)$ is larger than 1.5 , and the edges of piles are identified at locations with $B^{\text {eff }}=0.1$. The distances are calculated at 4 depths of $5,20,40 \mathrm{~km}$ and $68 \mathrm{~km}$ above the $\mathrm{CMB}$

(Supplementary Movie 1). We compute the lateral distance of hottest regions from the closest edges of thermochemical piles throughout the model run. We exclude the hottest regions that are within $500 \mathrm{~km}$ from side boundaries of the model domain. We find that the distances between the hottest regions and the edges of thermochemical piles range from a minimum of 100 's $\mathrm{km}$ to over $1500 \mathrm{~km}$, with a peak at around 500-1000 km (Fig. 2).

ULVZs caused by ultradense material. A second set of experiments considers the dynamics and evolution of ultradense material as a cause of ULVZs (Case 2). These experiments have three compositions: background mantle, thermochemical piles, and a small volume of ultradense material with a buoyancy number of $B_{\mathrm{u}}=2.0$ (or $9 \%$ denser than background mantle if scaled using reference parameters given in Supplementary Table 2). The ultradense material is initially introduced as a ubiquitous uniform layer in the lowermost $5 \mathrm{~km}$ of the mantle, and it quickly advects toward the pile edges, accumulating into discontinuous patches of varying size and shape (Supplementary Movie 2). Figure 3 shows a snapshot of this case at 227 Myr. Figure 3a displays the distribution of ultradense ULVZ material (red isosurfaces) underneath the thermochemical piles (partially transparent green isosurfaces). The accumulations vary in size from $\sim 100$ to $\sim 1000 \mathrm{~km}$ across and $\sim 5-100 \mathrm{~km}$ thick and have either rounded or linear map-view morphologies (discussed later). An interesting point to note is that the accumulations form into discontinuous patches, as opposed to ubiquitous, continuous ribbons along pile edges implied from $2 \mathrm{D}$ studies $^{9}$. Figure $3 \mathrm{~b}$ is a zoom-in of Fig. 3a that displays the effective buoyancy ratio in regions with ultradense material $5 \mathrm{~km}$ above the $\mathrm{CMB}$, illustrating the heterogeneity of density within the accumulations, caused by stirring with the surrounding mantle. Figure $3 c$ demonstrates that accumulations of ultradense material are typically quite thin, except for small regions within particularly large accumulations, where local heights may reach up to $100 \mathrm{~km}$ above the CMB. Figure $3 \mathrm{~d}-\mathrm{f}$ illustrates the variability in cross-sectional shape of the accumulations.
The lateral width of the accumulations greatly varies from place to place, and the cross-sectional shape of the accumulations is asymmetrical, thicker on the side in contact with the background mantle. This asymmetrical shape is due to differential viscous coupling, as noted in a previous $2 \mathrm{D}$ study ${ }^{9}$.

Figure 4 shows the compositional field at $5 \mathrm{~km}$ above the $\mathrm{CMB}$ for a time sequence of snapshots for Case 2, illustrating the time-dependence of the distribution of ultradense material. At 121 Myr (Fig. 4a), two large patches of ultradense material (labeled U1 and U2) are located at the edge of the pile. At 160 Myr (Fig. 4b), U2 has been advected into a linear shape, whereas U1 has maintained its rounded shape. At 227 Myr (Fig. 4c), U2 has split into three parts: a remnant of U2 (still labeled as U2) migrated toward U1, another formed into a smaller accumulation with relatively rounder shape (labeled U3), and another had been entrained into the pile, up along its side, and back down again (U4). In Fig. 4c, the ultradense material in U2 and U4 has experienced higher degree of stirring with pile material, leading to a lower effective buoyancy ratio (i.e., effective intrinsic density) in these patches than $\mathrm{U} 1$ and $\mathrm{U} 3$. In general, we observe that regions of long-term, stable, horizontally convergent mantle flow produces longer-lived, rounded accumulations of ultradense material. In contrast, linear accumulations are the result of ultradense material on the move, toward a location of more-stable convergent flow. Thus, ULVZ shape can change over time scales as short as tens of Myr.

Similar to Case 1, we compute the lateral distance of regions with accumulations of ultradense material from the closest edges of thermochemical piles for Case 2 (Fig. 2). We compute the distances throughout the model run but we exclude the first 50 Myr for Case 2 when the initial global layer of ultradense material is advecting to the edges of piles. We also exclude the regions with accumulations of ultradense material that are within $500 \mathrm{~km}$ from side boundaries of the model domain. In contrast to the wide range of distances between hottest regions and pile edges, the compositionally distinct ultradense material generally accumulates along the edges of thermochemical piles. At depths of 40 and $68 \mathrm{~km}$ above the CMB, the majority of ultradense materials occurs within $\sim 300 \mathrm{~km}$ from the pile edges (Fig. 2). At depths of 5 and $20 \mathrm{~km}$ above the CMB, the patches of ultradense material become much larger than at shallower depths (Fig. 3d), which leads to a significant amount of ultradense materials occurring between $\sim 300-800 \mathrm{~km}$ from the pile edges. However, even at depths of 5 and $20 \mathrm{~km}$ above the CMB, the largest fraction of ultradense materials still occurs within $\sim 300 \mathrm{~km}$ from pile edges.

Results of other geodynamic models. We also explored different combinations of parameters, including intrinsic density, volume and an intrinsic compositional viscosity decrease of the ultradense material, temperature-dependence of viscosity, Rayleigh number, and intrinsic density of thermochemical piles. Varying these parameters leads only to second-order differences from Case 1 or Case 2, but the fundamental conclusions about the distribution and accumulation of ultradense material, and the locations of hottest regions remain unchanged. We find that increasing the intrinsic density (Case 3 ) or initial volume (Case 4) of ultradense material acts to increase the size of the accumulations of ultradense material. Reducing the intrinsic density of ultradense material (Case 5) leads to more stirring between the ultradense material and pile material than Case 2. Using a larger activation coefficient $(A=11.51)$ for the temperature-dependent viscosity in Case 6 results in a slightly increase of the size of accumulations ultradense material. Similar to previous geodynamic modeling results ${ }^{44,45}$, we find that the morphology of 
a

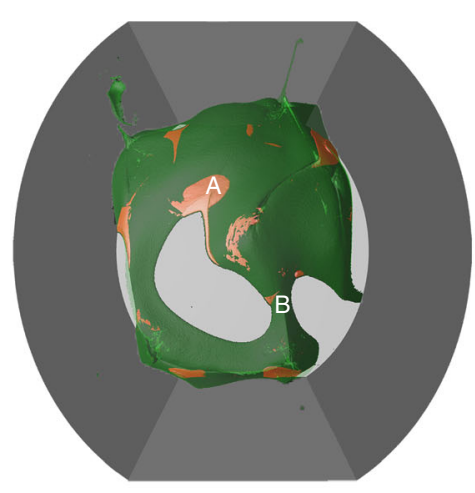

d

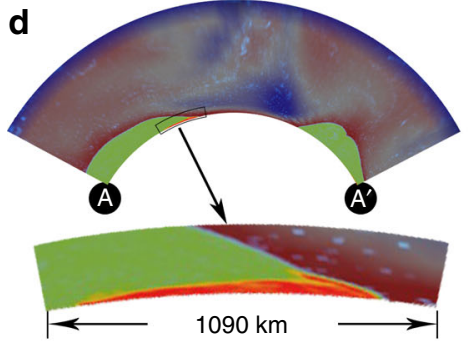

b

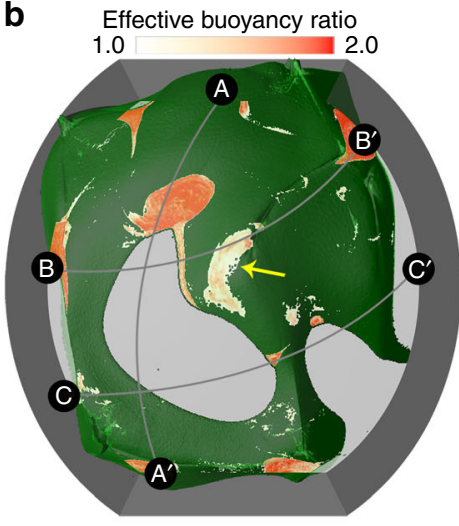

e

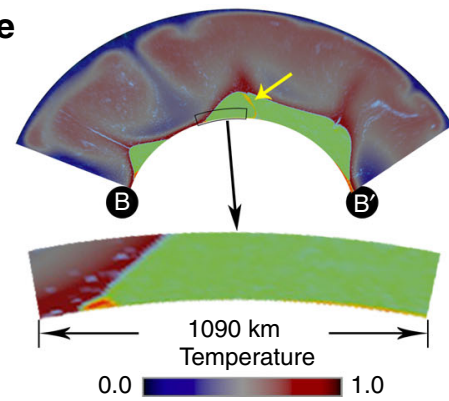

C Thickness of ultra-dense material $(\mathrm{km})$

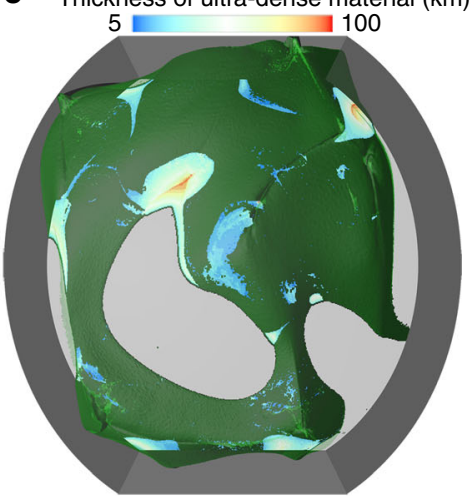

$\mathbf{f}$

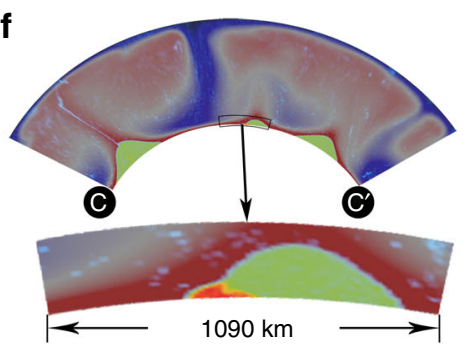

Fig. 3 Morphology and distribution ULVZs caused by compositionally distinct ultradense material. a Distribution of ultradense material (red isosurface) beneath large-scale thermochemical piles (partially transparent green isosurface). The lateral length-scale of accumulations of ultradense material at locations $A$ and $B$ is $~ 1000$ and $\sim 100 \mathrm{~km}$, respectively. The exposed Earth's core in downwelling regions is shown by light gray color. b A zoom-in of panel a along with the locations of 3 cross-sections. The effective buoyancy ratio field at $5 \mathrm{~km}$ above $\mathrm{CMB}$ show the locations of ultradense material (white to red color). The yellow arrow points to the region with relatively low-effective buoyancy ratio (whitish color) caused by stirring of ultradense material with pile material. c Same as in $\mathbf{b}$ but with the thickness of accumulations of ultradense material shown. $\mathbf{d}$-f $\mathbf{f}$ Cross-section at locations marked by gray lines in $\mathbf{b}$. The temperature field is shown by dark blue to red color, the thermochemical piles are shown in green color in the lowermost mantle and the ultradense material is shown by orange-red color at the base of piles. The yellow arrow in e points to ultradense material that has been viscously entrained up to the top of the pile along the pile edges, and later sank to the bottom of the pile. Beneath each panel is a zoomed-in region outlined by the black box at the edges of piles. All panels a-f are shown at $227 \mathrm{Myr}$

thermochemical piles is affected by using a different Rayleigh number, temperature-dependence of viscosity, and intrinsic density of thermochemical piles (Cases 7-11). However, for all cases discussed above, the distribution of ultradense material is similar to that in Case 2, with the majority of ultradense materials forming into discontinuous patches at the edges of thermochemical piles (Supplementary Figs. 3-8), and the hottest lowermost mantle regions are generally located well within the interiors of thermochemical piles (Supplementary Figs. 9-10). A more detailed discussion about the modeling results for Cases $3-11$ is provided in the Supplementary Note 2.

One caveat is that we do not include viscous dissipation in our models, so we do not have shear heating in the piles, which we consider negligible given their low viscosities. However, it is not inconceivable that certain combinations of material properties could lead to viscous heating, and therefore possible partial melting in other parts of the pile as well, such as near the edges where flow is changing direction.

Comparison with seismic observations of ULVZs. We show in Fig. 5a the seismic shear-wave tomography model S40RTS near the $\mathrm{CMB}^{46}$, with the edges of LLVPs marked by orange contours. We plot observations of ULVZs together with the edges of LLVPs in Fig. 5b. Here, we only select studies of ULVZs using core reflected waves $(\mathrm{ScS}, \mathrm{PcP}, \mathrm{ScP})$, in which the locations of ULVZs have minimum uncertainties (in comparison to the core waves or long path diffracted waves). The lateral size of the ULVZs is computed based on 1/4 wavelength Fresnel zones of the CMB reflection location for the waves used in each study. A list of references for these ULVZ observations is provided in the Supplementary Table 3 and Supplementary References.

As shown in Fig. 5b and also summarized in the Fig. 1 of ref. ${ }^{9}$, the ULVZs exhibit a variety of shapes and sizes, similar to the accumulations of ultradense material as labeled U1-4 in Fig. 4. For example, a larger-than-average, rounded ULVZ is observed near the north edges of the Pacific LLVP (Fig. 5b) and beneath Hawaii ${ }^{7}$, not unlike the large rounded U1 (Fig. 4); a linear shape ULVZ detected in the SW Pacific ${ }^{8}$ may be similar to the long linear U2 in Fig. 4b, c; and the ULVZs with small lateral-scale detected in many regions are analogous to the small U3. Our results show variable degrees of stirring of ultradense material with pile material (U2 and U4 compared to U1 and U3 in Fig. 4c), which may be analogous to the variable density increases observed in ULVZs ${ }^{3}, 4$. Our geodynamic modeling results also suggest that the accumulations of ultradense material are not ubiquitous along pile edges but form into discontinuous patches with variable morphology, demonstrating that not all LLVP margins are expected to contain ULVZs. This is supported by a recent detection of intermittent and unevenly distribution ULVZs at the northeastern margin of the Pacific LLVP ${ }^{47}$.

Similar to that shown in Fig. 2, we compute the closest lateral distances of observed ULVZs (as shown in Fig. 5b) to the edges of LLVPs along the CMB (Methods). Figure $5 \mathrm{c}$ shows that $55.5 \%$ of the computed ULVZ area occurs outside of the LLVPs (denoted with negative distance) and $44.5 \%$ ULVZ area occurs within the LLVPs (denoted with positive distance). We find that most 
a

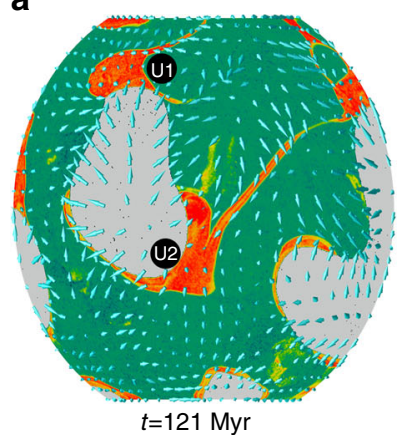

b

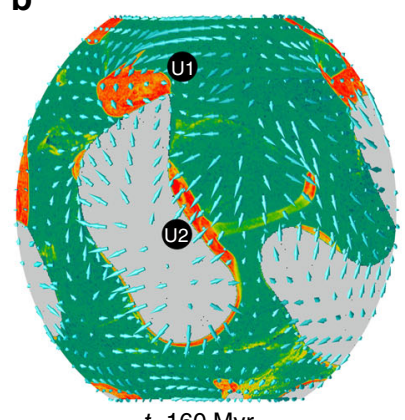

$t=160 \mathrm{Myr}$ c

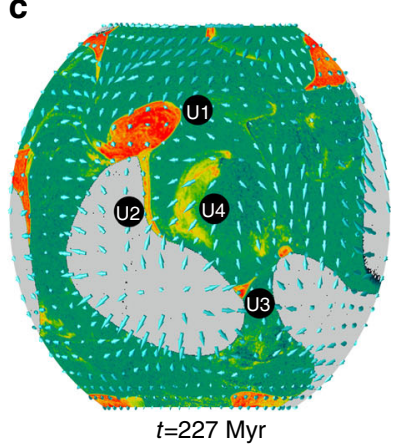

Effective buoyancy ratio

$0.1 \square 2.0$

Velocity magnitude $=1000$ (non-dimensional)

Fig. 4 Time evolution of the location and morphology of ultradense material patches. a-c Composition field (represented by effective buoyancy ratio) at $5 \mathrm{~km}$ above the CMB showing locations of ultradense material (reddish) and pile material (greenish). The yellowish color shows a mixing of ultradense material with pile material. The cyan arrows show mantle flow velocity. U1, U2, U3, and U4 are markers that track the patches of ultradense material, as explained in the text. Light gray color represents Earth's core

ULVZs are in close proximity of LLVP margins (Fig. 5c). Outside of LLVPs, almost all the ULVZ area occurs within $800 \mathrm{~km}$ from the edges of LLVPs. Inside of LLVPs, we find that the ULVZ area decreases linearly with the increase of distance to LLVP edges, and there is no ULVZ area occurring more than $1200 \mathrm{~km}$ from the LLVP edges.

We calculated the distance of ULVZs to the edges of LLVPs for other five tomography models (Supplementary Fig. 11). The amount of ULVZ area outside and inside of LLVPs differs somewhat between models, since the locations of the LLVP edges slightly differ among models. However, the general conclusion holds that ULVZs are both outside and inside the LLVPs, with most ULVZ area occurring within $\sim 800 \mathrm{~km}$ from LLVP edges of the tomographic models tested. The proximity of the observed ULVZs near LLVP edges is similar to the ultradense materials in our geodynamic models occurring near the edges of thermochemical piles (Fig. 2), suggesting a compositionally distinct component to ULVZs.

\section{Discussion}

The seismically derived ULVZs studied here have variable lateral dimensions, morphologies, and locations, consistent with a compositionally distinct origin of ULVZs. For the Earth, the crystallization of basal magma oceanic may initially produce a thin layer of ultradense material on the $\mathrm{CMB}^{29,48}$. The ultradense materials may be produced by the interaction between the core and the mantle and they may be produced at any location where the core and mantle interact ${ }^{23}$, 24 . In addition, the subduction of slabs may bring some intrinsically dense materials to the lowermost mantle outside of the LLVPs ${ }^{19-22}$. Our experiments are geared toward understanding thermochemical convection at equilibrium conditions; however, our model setup also allows us to explore (in a limited manner) how ultradense material gets swept from the surrounding mantle to the edges of thermochemical piles. Because our initial condition consists of a thin, uniform ultradense layer ubiquitous along the $\mathrm{CMB}$, the early times of the calculation exhibit the sweeping of this material toward the piles (Supplementary Movie 2; Supplementary Fig. 12). It demonstrates that any high-density compositional heterogeneity outside of piles is being advected toward the global upwelling regions (where the piles exist). Therefore, if ULVZs are caused by ultradense subduction remnants ${ }^{19-22}$ or core-mantle boundary reaction products ${ }^{23}$, 24 , we expect to observe them outside of piles as they are being advected toward them. Note that if compositional ULVZs have a lower solidus than background mantle, they may also include partial melt.

Though our results suggest that ULVZs located outside or at the edges of LLVPs are compositionally distinct from their surroundings, a small number of ULVZs located well within LLVPs ${ }^{9}$ (Fig. 5b) may be caused solely by partial melting. Interestingly, partial melting within the LLVPs would likely alter composition $^{49}$, perhaps producing a source of intrinsically dense heterogeneity with lower melting temperature $e^{49-51}$ that would continually advect toward LLVP edges.

\section{Methods}

Numerical modeling. We perform high-resolution three-dimensional calculations to investigate the morphology, distribution and dynamics of ULVZs by solving the following non-dimensional equations for conservation of mass, momentum, and energy under the Boussinesq approximation:

$$
\begin{gathered}
\nabla \cdot \mathbf{u}=0 \\
-\nabla P+\nabla \cdot(\eta \dot{\epsilon})=\xi R a\left(T-B^{\mathrm{eff}}\right) \mathbf{r} \\
\frac{\partial T}{\partial t}+(\mathbf{u} \cdot \nabla) T=\nabla^{2} T+H
\end{gathered}
$$

where, $\mathbf{u}$ is the velocity, $P$ is the dynamic pressure, $\eta$ is the viscosity, $\dot{\epsilon}$ is the strain rate tensor, $T$ is the temperature, $\mathbf{r}$ is the unit vector in radial direction, $B^{\text {eff }}$ is the effective buoyancy ratio (defined below). $t$ is time, and $H$ is internal heating. $\xi=\left(R_{\mathrm{e}} / D\right)^{3}$ with $R_{\mathrm{e}}$ as the Earth's radius and $D$ as the mantle thickness. Physical parameters in the above equations are all non-dimensional. The Eqs. (1)-(3) are solved using the CitcomCU code, which is available at https:/geodynamics.org/cig/ software/citcomcu/.

The thermal Rayleigh number $\mathrm{Ra}$ is defined as:

$$
\mathrm{Ra}=\frac{\rho_{0} g \alpha_{0} \Delta T D^{3}}{\eta_{0} \kappa_{0}}
$$

where $\rho_{0}, \alpha_{0}, \Delta T, \eta_{0}, \kappa_{0}$ are dimensional reference values of background mantle reference density, thermal expansivity, temperature difference between core-mantle boundary and surface, reference viscosity at temperature $T=0.6$ (non-dimensional), and thermal diffusivity, respectively. $g$ is dimensional gravitational acceleration.

The internal heating $H$ is non-dimensionalized as:

$$
H=\frac{R_{\mathrm{e}}^{2}}{\kappa_{0} c_{P_{0}} \Delta T} H^{*}
$$

where, $c_{\mathrm{P}_{0}}$ is heat capacity, $H^{*}$ is the dimensional heat production rate.

The buoyancy number for a compositional component $\left(B_{i}\right)$ is defined as the ratio between intrinsic density anomaly and density anomaly due to thermal expansion:

$$
B_{i}=\frac{\Delta \rho_{i}}{\rho_{0} \alpha_{0} \Delta T}
$$


a

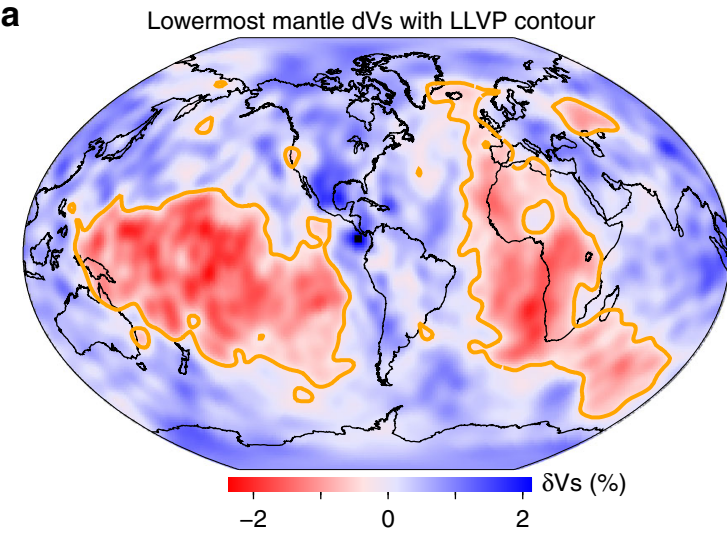

b

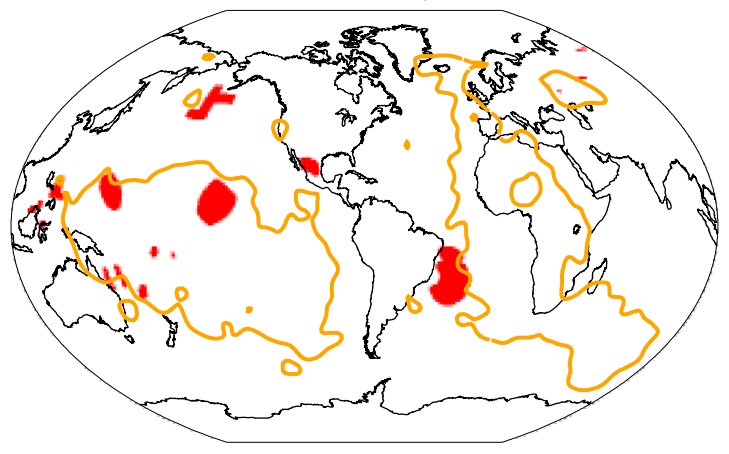

C

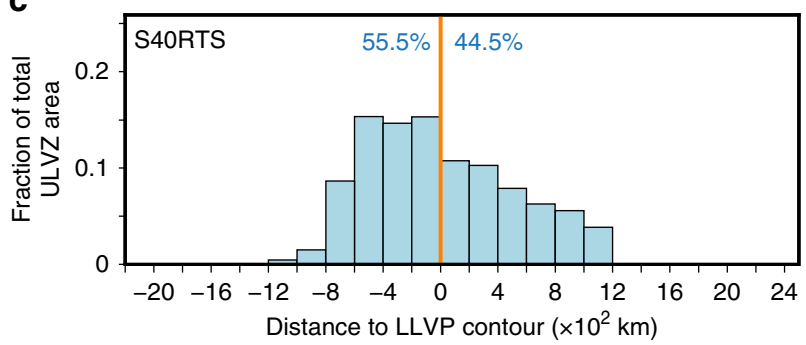

Fig. 5 Seismic observation of ULVZs and LLVP edges. a Seismic shear-wave tomography model at $2800 \mathrm{~km}$ depth (S4ORTS), with the LLVP edges shown by orange lines that surround $30 \%$ of the CMB area. b LLVP edges (orange lines) along with the ULVZ Fresnel zone patches (red, discretized in $0.5 \times 0.5$ degree cells) for all ULVZ waveform studies of core reflected waves (ScS, ScP, PcP). c The minimum distance of every ULVZ cell to LLVP edges. For each distance in the horizontal axis, the corresponding vertical axis shows the areal fraction of total ULVZ area. The thick orange line denotes the LLVP margin. Negative distance represents outside of LLVPs and positive distance represents inside of LLVPs. The percent area outside vs. inside of LLVPs is indicated by the blue text

where, $\Delta \rho_{i}$ is intrinsic density difference between an individual compositional component and the background mantle.

Similarly, the effective buoyancy ratio is defined as:

$$
B^{\mathrm{eff}}=\frac{\Delta \rho_{\mathrm{el}}}{\rho_{0} \alpha_{0} \Delta T}
$$

where $\Delta \rho_{\mathrm{el}}$ is effective intrinsic density anomaly on an element computed from the intrinsic density anomaly and the fraction of each compositional component in the element using the hybrid tracer method described below.

Hybrid tracer method. In thermochemical geodynamical modeling, two methods are typically used to model the advection of compositional field: the ratio tracer method and absolute tracer method ${ }^{37}$.

In the absolute tracer method, the composition fraction $\left(C_{i}\right)$ of each compositional component (except the background mantle) is proportional to the number of tracers per volume:

$$
C_{i}=\frac{N_{i} V_{0}}{V}
$$

where $N_{i}$ is the number of tracers for the $i$ th compositional component in an element, $V$ is the volume of the element and $V_{0}$ is a constant which equals to average volume per tracer for the $i$ th compositional component. For background mantle, $N_{i}$ equals zero (i.e., no tracer in the element) and $C_{i}$ becomes zero. Thus, there is no need for additional tracers to simulate the background mantle. This becomes a big advantage when the volume of chemical heterogeneities is very small (e.g., the ULVZs), which could be efficiently simulated with a small amount of tracers.

For ratio tracer method, the background mantle is also represented by tracers. Usually, the density of the background mantle is the reference density and the buoyancy number for the background mantle equals zero. The compositional fraction $\left(C_{i}\right)$ for each compositional component within an element is:

$$
C_{i}=\frac{N_{i}}{N}
$$

where $N_{i}$ is the number of tracers in the element used to simulate the $i$ th compositional component. $N$ is the total number of tracers in that element.

The ratio tracer method is benchmarked, and compared with absolute tracer method in ref. ${ }^{37}$. The ratio tracer method has several advantages over the absolute tracer method, such as minimal numerical diffusion and low entrainment. Thus, ratio tracer method is often used when dealing with large-scale chemical heterogeneities (i.e., LLVPs), because in this case the absolute tracer method also needs large amount of tracers and no longer has the advantage of modeling the compositional heterogeneities using less tracers.

In this study, our model is featured by both large-scale thermochemical piles and small-scale accumulations of ultradense material. We developed a hybrid tracer method which combines the advantages of ratio and absolute tracer method. Here, the background mantle and large scale compositional heterogeneities of piles are represented by $\sim 710$ million ratio tracers and the smaller scale accumulations of compositionally distinct ultradense material are simulated by $\sim 52-110$ million absolute traces (depending on the initial volume of ultradense material).

The effective intrinsic density anomaly $\left(\Delta \rho_{\mathrm{el}}\right)$ for each element in the computation domain contains two parts. One part is from background mantle and pile material which are modeled with ratio tracers, and is given by:

$$
\Delta \rho_{\mathrm{el}}^{\mathrm{r}}=\Delta \rho_{\mathrm{p}} C_{\mathrm{p}}+\Delta \rho_{\mathrm{bg}} C_{\mathrm{bg}}
$$

where, $\Delta \rho_{\mathrm{p}}$ is the intrinsic density anomaly of pile material. $C_{\mathrm{p}}$ is compositional fraction of pile material for the element which is calculated using Eq. (9). $\Delta \rho_{\mathrm{bg}}$ and $C_{\mathrm{bg}}$ are the intrinsic density anomaly and compositional fraction for the background mantle for the element, respectively. The intrinsic density anomaly of the background mantle is zero, so Eq. (10) becomes:

$$
\Delta \rho_{\mathrm{el}}^{\mathrm{r}}=\Delta \rho_{\mathrm{p}} C_{\mathrm{p}}
$$

The other part of the effective intrinsic density anomaly on an element $\left(\Delta \rho_{\mathrm{el}}\right)$ is from ultradense (i.e., ULVZ) material which is modeled with absolute tracers, and is given as:

$$
\Delta \rho_{\mathrm{el}}^{\mathrm{a}}=\Delta \rho_{\mathrm{u}} C_{\mathrm{u}}
$$

where $\Delta \rho_{\mathrm{u}}$ is the intrinsic density anomaly of ultradense material. $C_{\mathrm{u}}$ is compositional fraction of ultradense material for the element which is calculated using Eq. (8). We truncated $C_{\mathrm{u}}$ at 1 to avoid unphysically settling of tracers ${ }^{37}$.

In the hybrid tracer method, the effective intrinsic density anomaly on an element of the computational domain $\left(\Delta \rho_{\mathrm{el}}\right)$ is given by:

$$
\Delta \rho_{\mathrm{el}}=\Delta \rho_{\mathrm{el}}^{\mathrm{a}}+\Delta \rho_{\mathrm{el}}^{\mathrm{r}}\left(1-C_{\mathrm{u}}\right)
$$

or,

$$
\Delta \rho_{\mathrm{el}}=\Delta \rho_{\mathrm{u}} C_{\mathrm{u}}+\Delta \rho_{\mathrm{p}} C_{\mathrm{p}}\left(1-C_{\mathrm{u}}\right)
$$

Notice that, for $C_{\mathrm{u}}=0$ (element has no ultradense material), $\Delta \rho_{\mathrm{el}}$ is equivalently calculated using the ratio tracer method; for $C_{\mathrm{u}}=1$ (element is saturated with ultradense material), $\Delta \rho_{\mathrm{el}}$ is equivalently calculated using the absolute tracer method.

The effective buoyancy ratio $\left(B^{\text {eff }}\right)$ on an element is related to the effective intrinsic density anomaly $\left(\Delta \rho_{\mathrm{el}}\right)$ on this element by:

$$
B^{\text {eff }}=\Delta \rho_{\mathrm{el}} /\left(\rho_{0} \alpha_{0} \Delta T\right)
$$

Core-reflected wave ULVZ studies. In this study, we survey ULVZ studies that utilized core-reflected energy waveform analyses, e.g., PcP, ScP, and ScS. These 
waves have the potential to detect ULVZ structure at the CMB reflection point location. This differs from studies using core waves, e.g., SPdKS which can have an uncertainty regarding mapping ULVZ structure at the core entrance or exit location of the path (similarly, PKP and PKKP have this ambiguity). Some Pdiff and Sdiff studies have evidence for ULVZs (e.g., refs. $7,{ }^{2}$ ). While these analyses indicate specific ULVZ locations, the long paths of the diffracted wave result in some uncertainty as to where along the path the ULVZ is located. For this reason, we investigate ULVZ proximity to LLVP edges with just the core-reflected data. The studies, regions, and wave type are given in Supplementary Table 3.

Each of the ULVZ Fresnel zones of the studies in Supplementary Table 3 was decimated onto a 0.5 deg by 0.5 deg grid, with the area computed for each cell. The minimum distance to the nearest LLVP boundary is computed for each cell, and the fraction of the total ULVZ area summed up as a function of that minimum distance. This is display in Fig. 5c, as well as in Supplementary Fig. 11 for six tomographic models. The models are S40RTS ${ }^{46}$, along with HMSL-S06 ${ }^{53}$, S362ANI ${ }^{54}$, SEMUCB-WM1 ${ }^{55}$, SP12RTS ${ }^{56}$, and GyPsum ${ }^{57}$. The LLVP boundary is chosen to be the contour that surrounds $30 \%$ of the CMB by area that has the lowest shear wave speeds in the tomography model ${ }^{12}$. The results for all the tomographic models are similar in that there is a significant area percentage of ULVZs located outside the LLVPs.

Data availability. The authors declare that all relevant data supporting the findings of this study are available within the article and its Supplementary Information file or available upon request. The code, CitcomCU, is available from https://geodynamics.org/cig/software/citcomcu/. The authors' specific version of the code is available upon request.

Received: 17 October 2016 Accepted: 9 June 2017

Published online: 02 August 2017

\section{References}

1. Garnero, E. J. \& Helmberger, D. V. Seismic detection of a thin laterally varying boundary layer at the base of the mantle beneath the central-Pacific. Geophys. Res. Lett. 23, 977-980 (1996).

2. Williams, Q., Revenaugh, J. \& Garnero, E. A correlation between ultra-low basal velocities in the mantle and hot spots. Science 281, 546-549 (1998).

3. Idehara, K. Structural heterogeneity of an ultra-low-velocity zone beneath the Philippine Islands: Implications for core-mantle chemical interactions induced by massive partial melting at the bottom of the mantle. Phys. Earth Planet. Int 184, 80-90 (2011)

4. Rost, S., Garnero, E. J., Williams, Q. \& Manga, M. Seismological constraints on a possible plume root at the core-mantle boundary. Nature 435, 666-669 (2005).

5. Hutko, A. R., Lay, T. \& Revenaugh, J. Localized double-array stacking analysis of PcP: $\mathrm{D}^{\prime \prime}$ and ULVZ structure beneath the Cocos plate, Mexico, central Pacific, and north Pacific. Phys. Earth Planet. Int 173, 60-74 (2009).

6. Rondenay, S. \& Fischer, K. M. Constraints on localized core-mantle boundary structure from multichannel, broadband SKS coda analysis. J. Geophys. Res. 108, 2537 (2003).

7. Cottaar, S. \& Romanowicz, B. An unsually large ULVZ at the base of the mantle near Hawaii. Earth. Planet. Sci. Lett. 355-356, 213-222 (2012).

8. Thorne, M. S., Garnero, E. J., Jahnke, G., Igel, H. \& McNamara, A. K. Mega ultra low velocity zone and mantle flow. Earth Planet. Sci. Lett. 364, 59-67 (2013).

9. McNamara, A. K., Garnero, E. J. \& Rost, S. Tracking deep mantle reservoirs with ultra-low velocity zones. Earth Planet. Sci. Lett. 299, 1-9 (2010).

10. Dziewonski, A. M., Lekic, V. \& Romanowicz, B. A. Mantle anchor structure: An argument for bottom up tectonics. Earth. Planet. Sci. Lett. 299, 69-79 (2010).

11. Garnero, E. J. \& McNamara, A. K. Structure and dynamics of earth's lower mantle. Science 320, 626-628 (2008).

12. Garnero, E. J., McNamara, A. K. \& Shim, S.-H. Continent-sized anomalous zones with low seismic velocity at the base of Earth's mantle. Nat. Geosci. 9, 481-489 (2016).

13. He, Y. \& Wen, L. Geographic boundary of the "Pacific Anomaly" and its geometry and transitional structure in the north. J. Geophys. Res. 117, B09308 (2012).

14. Brown, S. P., Thorne, M. S., Miyagi, L. \& Rost, S. A compositional origin to ultralow-velocity zones. Geophys. Res. Lett. 42, 1039-1045 (2015).

15. Wicks, J. K., Jackson, J. M. \& Sturhahn, W. Very low sound velocities in ironrich $(\mathrm{Mg}, \mathrm{Fe}) \mathrm{O}$ : Implications for the core-mantle boundary region. Geophys. Res. Lett. 37, L15304 (2010).

16. Wicks, J., Jackson, J. M., Sturhahn, W. \& Zhang, D. Sound velocity and density of magnesiowüstites: Implications for ultralow-velocity zone topography. Geophys. Res. Lett. 44, 2148-2158 (2017).

17. Mao, W. L. et al. Iron-rich post-perovskite and the origin of ultralow-velocity zones. Science 312, 564-565 (2006).
18. Li, Y., Deschamps, F. \& Tackley, J. P. Small post-perovskite patches at the base of lower mantle primordial reservoirs: Insights from 2-D numerical modeling and implications for ULVZs. Geophys. Res. Lett. 43, 3215-3225 (2016).

19. Dobson, D. P. \& Brodholt, J. P. Subducted banded iron formations as a source of ultralow-velocity zones at the core-mantle boundary. Nature. 434, 371-374 (2005).

20. Andrault, D. et al. Melting of subducted basalt at the core-mantle boundary. Science 344, 892-895 (2014).

21. $\mathrm{Hu}, \mathrm{Q}$. et al. $\mathrm{FeO}_{2}$ and $\mathrm{FeOOH}$ under deep lower-mantle conditions and Earth's oxygen-hydrogen cycles. Nature 534, 241-244 (2016).

22. Liu, J., Li, J., Hrubiak, R. \& Smith, J. S. Origins of ultralow velocity zones through slab-derived metallic melt. Proc. Natl Acad. Sci. USA 113, 5547-5551 (2016).

23. Buffett, B. A., Garnero, E. J. \& Jeanloz, R. Sediments at the top of Earth's core. Science 290, 1338-1342 (2000).

24. Otsuka, K. \& Karato, S. Deep penetration of molten iron into the mantle caused by a morphological instability. Nature 492, 243-246 (2012).

25. Bower, D. J., Gurnis, M. \& Seton, M. Lower mantle structure from paleogeographically constrained dynamic Earth models. Geochem. Geophys. Geosyst. 14, 44-63 (2013).

26. McNamara, A. K. \& Zhong, S. Thermochemical structures beneath Africa and the Pacific Ocean. Nature 437, 1136-1139 (2005).

27. Zhang, N., Zhong, S., Leng, W. \& Li, Z.-X. A model for the evolution of the Earth's mantle structure since the Early Paleozoic. J. Geophys. Res. 115, B06401 (2010).

28. Deschamps, F., Cobden, L. \& Tackley, P. J. The primitive nature of large low shear-wave velocity provinces. Earth Planet. Sci. Lett. 349, 198-208 (2012).

29. Labrosse, S., Hernlund, J. W. \& Coltice, N. A crystallizing dense magma ocean at the base of the Earth's mantle. Nature. 450, 866-869 (2007).

30. Li, M., McNamara, A. K. \& Garnero, E. J. Chemical complexity of hotspots caused by cycling oceanic crust through mantle reservoirs. Nat. Geosci. 7, 366-370 (2014).

31. Wen, L. A compositional anomaly at the Earth's core-mantle boundary as an anchor to the relatively slowly moving surface hotspots and as source to the DUPAL anomaly. Earth. Planet. Sci. Lett. 246, 138-148 (2006).

32. Zhang, Z. et al. Primordial metallic melt in the deep mantle. Geophys. Res. Lett. 43, 3693-3699 (2016).

33. White, W. M. Isotopes, DUPAL, LLSVPs, and Anekantavada. Chem. Geol. 419, 10-28 (2015).

34. Williams, C. D., Li, M., McNamara, A. K., Garnero, E. J. \& van Soest, M. C. Episodic entrainment of deep primordial mantle material into ocean island basalts. Nat. Commun. 6, 8937 (2015).

35. Gu, T., Li, M., McCammon, C. \& Lee, K. K. M. Redox-induced lower mantle density contrast and effect on mantle structure and primitive oxygen. Nat. Geosci. 9, 723-727 (2016).

36. Zhong, S. Constraints on thermochemical convection of the mantle from plume heat flux, plume excess temperature, and upper mantle temperature. J. Geophys. Res. 111, B04409 (2006).

37. Tackley, P. J. \& King, S. D. Testing the tracer ratio method for modeling active compositional fields in mantle convection simulations. Geochem. Geophys. Geosyst. 4, 8302 (2003).

38. Andrault, D. et al. Solidus and liquidus profiles of chondritic mantle: Implication for melting of the Earth across its history. Earth Planet. Sci. Lett. 304, 251-259 (2011).

39. Fiquet, G. et al. Melting of peridotite to 140 gigapascals. Science 329, 1516-1518 (2010).

40. Nomura, R. et al. Low core-mantle boundary temperature inferred from the solidus of pyrolite. Science 343, 522-525 (2014).

41. Lay, T., Hernlund, J. \& Buffett, B. A. Core-mantle boundary heat flow. Nat. Geosci. 1, 25-32 (2008).

42. Christensen, U. R. \& Hofmann, A. W. Segregation of subducted oceanic crust in the convecting mantle. J. Geophys. Res. 99, 19867-19884 (1994).

43. Li, M. \& McNamara, A. K. The difficulty for subducted oceanic crust to accumulate at the Earth's core-mantle boundary. J. Geophys. Res. 118, 1807-1816 (2013).

44. Deschamps, F. \& Tackley, P. J. Searching for models of thermo-chemical convection that explain probabilistic tomography. Phys. Earth Planet. Int 171, 357-373 (2008).

45. Deschamps, F. \& Tackley, P. J. Searching for models of thermo-chemical convection that explain probabilistic tomography. II-Influence of physical and compositional parameters. Phys. Earth Planet. Int 176, 1-18 (2009).

46. Ritsema, J., Deuss, A., van Heijst, H. J. \& Woodhouse, J. H. S40RTS: a degree-40 shear-velocity model for the mantle from new Rayleigh wave dispersion, teleseismic traveltime and normal-mode splitting function measurements. Geophys. J. Int 184, 1223-1236 (2011).

47. Zhao, C., Garnero, E. J., Li, M., McNamara, A. \& Yu, S. Intermittent and lateral varying ULVZ structure at the northeastern margin of the Pacific LLSVP. J. Geophys. Res. 122, 1198-1220 (2017). 
48. Boukaré, C. E., Ricard, Y. \& Fiquet, G. Thermodynamics of the MgO-FeO-SiO system up to $140 \mathrm{GPa}$ : Application to the crystallization of Earth's magma ocean. J. Geophys. Res. 120, 6085-6101 (2015).

49. Nomura, R. et al. Spin crossover and iron-rich silicate melt in the Earth's deep mantle. Nature. 473, 199-202 (2011).

50. Boehler, R. Melting of the Fe-FeO and the Fe-FeS systems at high pressure: Constraints on core temperatures. Earth. Planet. Sci. Lett. 111, 217-227 (1992).

51. Zerr, A. \& Boehler, R. Constraints on the melting temperature of the lower mantle from high-pressure experiments on $\mathrm{MgO}$ and magnesioustite. Nature. 371, 506-508 (1994).

52. Xu, Y. \& Koper, K. D. Detection of a ULVZ at the base of the mantle beneath the northwest Pacific. Geophys. Res. Lett. 36, L17301 (2009).

53. Houser, C., Masters, G., Shearer, P. \& Laske, G. Shear and compressional velocity models of the mantle from cluster analysis of long-period waveforms. Geophys. J. Int. 174, 195-212 (2008).

54. Kustowski, B., Ekström, G. \& Dziewoński, A. M. Anisotropic shear-wave velocity structure of the Earth's mantle: A global model. J. Geophys. Res. 113, B06306 (2008).

55. French, S. W. \& Romanowicz, B. A. Whole-mantle radially anisotropic shear velocity structure from spectral-element waveform tomography. Geophys. J. Int. 199, 1303-1327 (2014).

56. Koelemeijer, P., Ritsema, J., Deuss, A. \& van Heijst, H. J. SP12RTS: a degree-12 model of shear- and compressional-wave velocity for Earth's mantle. Geophys. J. Int. 204, 1024-1039 (2016).

57. Simmons, N. A., Forte, A. M., Boschi, L. \& Grand, S. P. GyPSuM: A joint tomographic model of mantle density and seismic wave speeds. J. Geophys. Res. 115, B12310 (2010).

\section{Acknowledgements}

We thank F. Deschamps, S. King and an anonymous reviwer for their constructive comments. The project is supported by NSF grants EAR-1045788, EAR-1401270 and EAR-1648817.

\section{Author contributions}

M.L., A.K.M. and E.J.G. contributed to conceiving the idea. M.L. carried out the numerical calculation. A.K.M. supervised the project. S.Y. and E.J.G. reviewed previous studies on ULVZs and digitized the location and size of ULVZs. All authors contributed to writing the paper.

\section{Additional information}

Supplementary Information accompanies this paper at doi:10.1038/s41467-017-00219-x.

Competing interests: The authors declare no competing financial interests.

Reprints and permission information is available online at http://npg.nature.com/ reprintsandpermissions/

Publisher's note: Springer Nature remains neutral with regard to jurisdictional claims in published maps and institutional affiliations.

\begin{abstract}
(c) (i) Open Access This article is licensed under a Creative Commons Attribution 4.0 International License, which permits use, sharing, adaptation, distribution and reproduction in any medium or format, as long as you give appropriate credit to the original author(s) and the source, provide a link to the Creative Commons license, and indicate if changes were made. The images or other third party material in this article are included in the article's Creative Commons license, unless indicated otherwise in a credit line to the material. If material is not included in the article's Creative Commons license and your intended use is not permitted by statutory regulation or exceeds the permitted use, you will need to obtain permission directly from the copyright holder. To view a copy of this license, visit http://creativecommons.org/ licenses/by/4.0/.
\end{abstract}

(c) The Author(s) 2017 